\title{
Integrated Optics: a Report on the 2nd OSA Topical Meeting
}

\author{
R. V. Pole, E. M. Conwell, H. Kogelnik, P. K. Tien, J. R. Whinnery, \\ A. Yariv, and A. J. De Maria
}

This report surveys the papers presented at the 2nd OSA Topical Meeting on Integrated Optics, which was held 21-24 January 1974 in New Orleans, La.

\section{Foreword}

\section{R. V. Pole}

This report surveys the papers presented at the 2nd Topical Meeting on Integrated Optics, which was held on 21-24 January 1974 in New Orleans, La. The meeting was sponsored by the Lasers and Electro-Optics Technical Group of the Optical Society of America, the Quantum Electronics Council of the Institute of Electrical and Electronics/Engineers, and the International Commission for Optics.

The 1st Topical Meeting on Integrated Optics was held some two years prior to this one, in Las Vegas, Nev. While that first meeting-not much unlike the town itself-had been characterized by some sort of adolescent exuberance, the papers at this meeting portrayed integrated optics as a gradually maturing technology. Whereas in Las Vegas the progress in the field was measured by the number of novel device concepts, the progress in New Orleans had to be judged in terms of quantitative improvements of already established concepts and a deeper theoretical understanding of the processes involved. It is this type of progress that was evident in New Orleans.

\section{Fibers and Waveguides \\ R. V. Pole}

\section{Progress in Fibers}

The technical part of the meeting was opened by two invited papers. The first invited paper was by

R. V. Pole is with IBM Research Laboratory, San Jose, California 95193; E. M. Conwell is with Xerox Rochester Corporate Research Center, Rochester, New York 14644; H. Kogelnik and P. K. Tien are with Bell Telephone Laboratories, Holmdel, N. J. 07733; J. R. Whinnery is with Electronics Research Laboratory, University of California, Berkeley, California 94720; A. Yariv is with California Institute of Technology, Pasadena, California 91109; A. J. De Maria is with United Aircraft Research Laboratories, East Hartford, Connecticut 06108.

Received 18 September 1974.
Robert D. Maurer [MA1] of Corning Glass Works, one of the pioneers in the evolution of the modern optical fibers, who surveyed the state of the art.

While recent years have witnessed marked advances in materials preparation and fabrication of optical waveguides, the battle has not yet been fully won. The initial requirements on low attenuation have been augmented by considerations such as low material cost, low intrinsic scattering, low intrinsic absorbtion, easy formability, low dispersion, low temperature dependence, insensitivity to background radiation, etc. Some of these considerations have barely bein touched, while others have received only preliminary attention. Nevertheless, some insight may be gained into how these demands have been met in four different glasses by referring to Table I.

The second invited paper by S. E. Miller [MA2] of Bell Laboratories, dealt more broadly with optical fibers as information transmission media and the status of this research. There are a number of types of optical fibers under study: (1) conventional core fiber, with a step change in refractive index at the core-cladding interface, (2) dielectric-tube fiber, (3) graded-index fiber and its special version, the parabolic-index fiber, and finally (5) the single-material fiber.

Two major parameters that determine the performance of a fiber are its loss and its delay distortion. Mechanisms causing loss are material absorption, material scattering, radiation loss at the curvatures, and losses in claddings. The delay distortion is caused by intrinsic material dispersion and the differential delay of various modes of a multimode fiber. While the former depends only on the material, the latter can be minimized by grading the transverse index distribution, by using losses selective among the modes, or by using intentional mode mixing.

There is no longer a question of whether fibers will be used for optical communications, but rather when it will occur. Since suitable lasers are still under development, initial communication links may use ei- 


\begin{tabular}{|c|c|c|c|c|}
\hline & Silica & Doped silica & $\begin{array}{l}\text { Thallium } \\
\text { borosilicate }\end{array}$ & Soda lime \\
\hline Intrinsic scattering $\left(\mathrm{dB} \mathrm{km}^{-1}\right)$ & 1.5 & $\sim 2$ & - & $\sim 2$ \\
\hline Intrinsic absorption ${ }^{a}$ & $\sim 0$ & $\sim 0$ & - & $<6$ \\
\hline Extraneous absorption and scattering & 2.5 & 2 & 一 & $\sim 30$ \\
\hline $\begin{array}{l}\text { Fiber attenuation at } 0.82 \mu \mathrm{m} \text { (sum) } \\
\qquad\left(\mathrm{dB} \mathrm{km}^{-1}\right)^{b}\end{array}$ & 4 & 4 & 20 & 38 \\
\hline Formability & Satisfactory & Satisfactory & Good & Good \\
\hline Material dispersion $\left(\sec ^{\dot{2}} \mathrm{~km}^{-1}\right)$. & $3 \times 10^{-23}$ & $4 \times 10^{-23 d}$ & 一 & $6 \times 10^{-23 d}$ \\
\hline $\begin{array}{l}\text { Radiation sensitivity }\left(\mathrm{dB} \mathrm{km}^{-1} \text { at }\right. \\
\quad 0.82 \mu \mathrm{m} \text { for } 20 \mathrm{rad})^{0}\end{array}$ & $<0.001^{f}$ & 0.03 & 28 & $0.15^{f}$ \\
\hline
\end{tabular}

a Estimated from analysis of attenuation in the lowest attenuation fibers made to date.

${ }^{b}$ Lowest attenuation fibers reported to date.

' Multiply by the waveguide length and source frequency width to get an approximate pulse width (all values at $0.82 \mu \mathrm{m}$ ).

${ }^{d}$ Estimated from similar glasses.

${ }^{e}$ Constancy of this data from fiber to fiber has not been established.

$f$ Determined in bulk glass.

ther LED's or lasers. In either case bandwidths up to $300 \mathrm{Mb} / \mathrm{sec}$ are possible.

First optical communication links will most probably be hybrid systems consisting of individual components such as distributed-feedback lasers, and gratings as wavelength filters, or structures for modulators. As the technology progresses, more overall integration is likely to take place.

Another detailed version of this interesting survey paper has been published elsewhere. ${ }^{1}$

\section{Thin-Film Waveguides}

The first contributed paper was by E. M. Conwell [MA3] of Xerox. In this theoretical paper she described the modes in anisotropic waveguides formed by diffusion, such as Se-diffused CdS and out-diffused $\mathrm{LiNbO}_{3}$.

Two other papers dealt with so-called rib and/or strip waveguides. These waveguides, long known in the field of microwave acoustics have apparently gained importance in integrated optics due to potentially low losses. They consist of strips of material on a slab substrate: the strip, generally of rectangular cross section, may have the same refractive index as the substrate (rib waveguide) or a higher one (strip-loaded waveguide).

The first of the two papers, by E. A. J. Marcatili [MA4] of Bell Laboratories, was a theoretical treatise of such guides or, more precisely, their simpler but closely equivalent form. The number of modes, their field distributions, and propagation constants were derived. Furthermore, requirements for simple mode operation, field penetration in the slab, and tolerances on the curvature of the guide were given.

The second paper, by F. K. Reinhart, R. A. Logan and T. P. Lee [MA5], also of Bell Laboratories, reported the transmission properties of an experimental rib waveguide formed by anodization of epitaxial GaAs on an $\mathrm{Al}_{x} \mathrm{Ga}_{1-x} \mathrm{As}$ layer. Their measurements were in excellent agreement with Marcati- li's theoretical results. The pictures of various lower order modes were impressive.

\section{Waveguides for Infrared Region}

The invited paper by P. K. Cheo [MA6] described various experiments conducted at the United Aircraft Research Laboratories using optical waveguides for the purpose of increasing the efficiency and reliability of eo modulators and deflectors in the $10-\mu \mathrm{m}$ region of the spectrum.

J. H. McFee, M. A. Pollack, W. W. Rigrod, and R. A. Logan [MA7] of Bell Laboratories described experiments in which they grew a GaAs guiding layer on a relatively low conductivity AlGaAs substrate with the hope of achieving low loss guides due to low conductivity of the substrate. They were partially successful.

The session was concluded by a pair of papers from an MIT group describing epitaxial growth of InGaAs on $\mathrm{GaAs}$ [MA8] and an integrated $\mathrm{In}_{x} \mathrm{Ga}_{1-x}$ As Shottky barrier waveguide deflector [MA9].

\section{Lasers \\ H. Kogelnik}

Comparing the presentations on thin-film lasers of the New Orleans meeting with those of the Las Vegas meeting of two years ago, one cannot fail to notice two points. The first is that interest has shifted from dye lasers to solid-state lasers, and in particular to semiconductor lasers, and the second is that a considerable materials and fabrication effort has been made during the past two years.

In a review of thin-film waveguide lassers C. V. Shank [MB1] of Bell Laboratories summarized the potential advantages (small size, low threshold, low heat dissipation and power consumption, stability, and compatibility with other waveguide devices) and potential disadvantages (stringent material requirements and need for a high density of active species, 
difficulties arising when optical pumping is needed) of applying optical guided-wave techniques to the construction of lasers. Shank gave an overview of the various thin-film laser material systems that have been studied ( $\mathrm{GaAs}$ heterostructure lasers, $\mathrm{Nd}^{+3}$ and $\mathrm{Ho}^{+3}$ solid-state lasers, and dye lasers), and pumping configurations (electrical pumping as well as transverse, longitudinal, and evanescent optical pumping) and resonator structures (cleaved edges, ring lasers, corner prisms, periodic structures, and distributed feedback) that have been used or proposed. Subsequently, he reported experiments done by C. V. Shank, R. V. Schmidt, and B. I. Miller on GaAlAs heterostructure lasers with distributed feedback. Here surface corrugations with a period of about $1000 \AA$ were fabricated in the GaAs layer by uv-laser exposure and ion-beam etching techniques, and a $\mathrm{Ga}_{0.5} \mathrm{Al}_{0.5} \mathrm{As}$ layer was regrown over this corrugation using a dummy seed-crystal technique to equilibrate the solution and eliminate backdissolving of the corrugation. These devices were pumped optically at $6400 \AA$ with a pulsed dye laser and output spectra less than $1 \AA$ in width were observed at $77 \mathrm{~K}$. In this connection it is interesting to note that two other groups reported successful regrowth of $\mathrm{Ga}(\mathrm{Al}) \mathrm{As}$ layers over corrugated surfaces. A. Yariv (Caltech) and co-workers [TuB9] used the uv-laser exposure but no dummy-crystal technique and consequently observed some backdissolving of the corrugation. L. Yang and co-workers (of Cornell University) as described in their postdeadline paper, used scanning electron beam exposure and the dummy-crystal technique. Both groups obtained evidence by visual inspection that successful regrowth over corrugations with a $3500-\AA$ periodicity had been achieved.

D. Schicketanz and C. Zeidler [MB2] of Siemens reported further experimental results on the use of GaAs double-heterostructure injection lasers as amplifiers. This work builds on earlier results, ${ }^{2}$ where the potential of broadband optical amplifiers for the repeaters of optical fiber communication systems is discussed. The authors have measured the gain, gain-saturation, and noise characteristics of these semiconductor lasers both as a function of pump current and wavelength. An amplification of $10 \mathrm{~dB}$ appears readily achievable. Regenerative amplification was also investigated using the reflections of the GaAs-air interface. In the regenerative mode gains of $20 \mathrm{~dB}$ can be obtained, but the saturation behavior shows a strong dependence on pump current, implying a need to stabilize the current to $\pm 1 \mathrm{~mA}$ and the temperature to $\pm 0.3 \mathrm{~K}$ in practical cases.

M. K. Barnoski et al. [MB3] of Hughes Research Laboratories reported a flexible ion-implantation technique for fabricating $p-n$ junction lasers. This also appears suitable for the production of distributed-feedback lasers. A dose of $10^{16} / \mathrm{cm}^{2} \mathrm{Zn}^{+}$ions is implanted at $20 \mathrm{kV}$ into a substrate of $n$-type GaAs doped with $10^{18} / \mathrm{cm}^{3}$ tellurium. The implantation forms a $p$-type layer with a thickness of about $1 \mu \mathrm{m}$. The junction depth can be accurately controled by varying the anneal time. The anneal cycle, which serves to electrically activate the $\mathrm{Zn}^{+}$ions and to remove damage caused by the implantation, was optimized to produce both good electrical characteristics and efficient optical emission. After the annealing, ohmic contacts are produced by Au:Ge eutectic metalization. Laser action in these devices was obtained at $77 \mathrm{~K}$ with 200 -nsec-wide current pulses at a $200-\mathrm{Hz}$ repetition rate. The smallest threshold current density observed was $2 \times 10^{3} \mathrm{~A} / \mathrm{cm}^{2}$.

W. C. Scott et al. [MB6] of Texas Instruments reported a new technique to fabricate a resonator structure in $\mathrm{GaAs}$ that does not require the cleaving of crystal edges. They masked a GaAs substrate with an oxide film having a diamond-shaped opening through which they grew a GaAs mesa layer by vapor phase epitaxy. This layer can be made $3-40 \mu \mathrm{m}$ high and provides sharp and aligned edges suitable to form a laser resonator. Laser action has been observed in these structures at $85 \mathrm{~K}$ by optical pumping at $100-\mathrm{kW}$ level.

M. Mawabe et al. [MB4] of Osaka University reported on the epitaxial growth of $\mathrm{CdS}_{1-x} \mathrm{Se}_{x}$ layers on $\mathrm{CdS}$ substrates. Vapor phase epitaxy was used, and layer thicknesses of typically $2 \mu \mathrm{m}$ were obtained. The suitability of this heterostructure as a thin-film laser was examined in experiments where a pulsed $\mathrm{N}_{2}$ laser was used as a pump. At $80 \mathrm{~K}$ laser action was observed near $5020 \AA$, and gain values up to $58 \mathrm{~cm}^{-1}$ were measured.

J. P. van der Ziel [MB5] of Bell Laboratories reported the observation of laser action in $\mathrm{Nd}^{3+}: \mathrm{YAG}$ waveguides. Thin epitaxial YAG films containing 2 mole percent $\mathrm{Nd}^{3+}$ were grown on YAG substrates with film thickness from $40 \mu \mathrm{m}$ to $250 \mu \mathrm{m}$. The presence of the $\mathrm{Nd}^{3+}$ increases the refractive index by 0.7 $\times 10^{-3}$. The films supported several guided modes, and the laser threshold for flashlamp pumping was comparable to the typical rod configuration. For longitudinal pumping with an argon laser at $5145 \AA$, continuous oscillation at $1.06 \mu \mathrm{m}$ was obtained with a pump threshold of $60 \mathrm{~mW}$.

D. Castleberry and co-workers [MB7] at MIT reported continuous single-mode operation at $2.06 \mu \mathrm{m}$ of a $\mathrm{Ho}^{3+}$ :YLF miniature laser sensitized by $\mathrm{Er}$ and $\mathrm{Tm}$. The laser was operated at $77 \mathrm{~K}$ and pumped with a cw krypton laser at $6471 \AA$. A threshold of about $20 \mathrm{~mW}$ of pump power was measured. Four sides of the crystal were polished, and the laser was operated in a total internal reflection mode. The laser light was coupled to a thin-film guide via a coupling gap.

H. P. Weber et al. [MB8] of Bell Laboratories reported their recent findings on the Nd pentaphosphate laser. Crystals with long fluorescent lifetimes $(0.2 \mathrm{msec})$ were obtained when the crystals were grown at temperatures above $600^{\circ} \mathrm{C}$ or when the crystals were annealed after growth at about $600^{\circ} \mathrm{C}$ in a $\mathrm{P}_{2} \mathrm{O}_{5}$ atmosphere. This way the authors achieved good crystal quality with $\mathrm{Nd}$ concentrations thirty times higher than in standard 1\% Nd:YAG. The dependence of the lifetime on $\mathrm{Nd}$ concentration was measured, and the concentration quenching pa- 

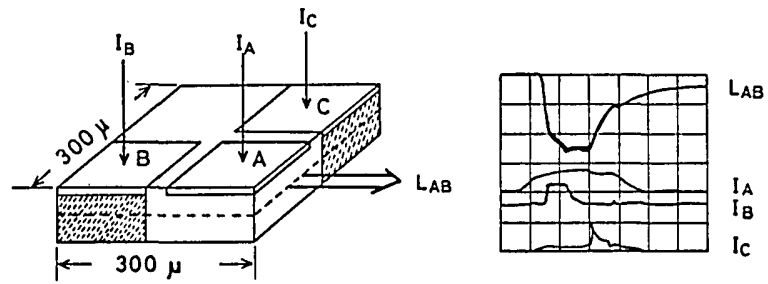

Fig. 1. Three-section laser diode described by Nishizawa and Ishida [WB2] and response with pulses applied to the three elements.

rameter was found to be a factor 7 smaller than that of Nd:YAG. By longitudinal pumping the workers obtained $\mathrm{cw}$ laser action in several crystal directions and for several pump lines of argon and krypton lasers. By the use of intracavity etalons the cw pentaphosphate lasers were made to operate at a single frequency. This last experiment indicated homogeneous line broadening, as almost all the available power was concentrated in the single output frequency.

P. Burlamacchi et al. [MB9] of Italy's CNR described an application of guided-wave techniques to high energy pulsed rhodamine 6G dye lassers. Here a dye cell of planar slab geometry is pumped from both sides by flashlamps. The absorption of the pump light leads to temperature gradients in the dye solution, which results in refractive index gradients suitable to form a slab waveguide. If the transverse dimension of the cell is made a few absorption lengths wide, one achieves a good overlap of the guided laser light and the inverted gain region in the dye solution. Overall efficiencies of $0.7 \%$ and energy densities of about $7 \mathrm{~J} / \mathrm{cm}^{2}$ were obtained with this configuration, which also provided good reproducibility from pulse to pulse, as the induced index gradients were much larger than any random perturbations due to shock or turbulence.

\section{Waveguides and Couplers}

\section{J. R. Whinnery}

\section{Military Uses of Integrated Optics}

In an invited paper, D. J. Albares [WB1] of the Naval Electronics Center and his colleagues described anticipated uses of film and integrated optics in the Department of Defense. In addition to the obvious potential for greater information capacity, he listed the following advantages:

(1) immunity to electromagnetic interference (EMI);

(2) freedom from electric short circuits and ground loops;

(3) safety in combustible areas;

(4) heat resistance;

(5) small size and light weight;

(6) less chance of theft, since glass not a strategic material;
(7) possible greater immunity to nuclear radiation. First applications seem most promising for aircraft because of the premium on saving weight. A prototype A-7D/E navigation and weapons delivery system is now being developed. Next emphasis will be on a data bus, suitable for both aircraft and shipboard use. Land-based use would take advantage of the immunity to EMI and the light weight for portable systems. The towed array, where weight is a serious problem, is a potential candidate for undersea applications. Preliminary estimates of savings to the Department of Defense by the use of fibers and thinfilm optics was $\$ 53 \times 10^{6}$ per year, primarily from savings in weight.

For data-bus applications, four-pole switching will be required. A prototype of a four-pole switch was described and forseeable improvements in multipole switching times were given.

\section{Thin-Film Optical Logic Devices}

Essentially the only paper of the conference concerned with logic operations was the invited paper by J. Nishizawa and K. Ishida [WB2] of Tohoku University concerned with the control of emission from one laser by light from another. Basic mechanisms are the amplification of the laser diode, the optical pumping effect, and the interaction through injected carriers. $^{3}$ The last of these is considered especially important for logic operations, since the injected carriers recombine in cooperation with a certain electromagnetic mode in proportion to the energy of that mode. ${ }^{3}$

After illustrating the effect of injected carriers on frequency response and time-domain characteristics for two interacting diodes, the response of a threediode arrangement on a single substrate was shown (Fig. 1). When common section laser diodes $A$ and $B$ are driven by a short switch pulse, a common mode between the two sections starts and continues lasing even after the end of the switch pulse. But if diode $C$ is now driven by a short pulse, it quenches the first oscillation, and a common mode between $A$ and $C$ starts to operate. In addition to the logic operations proposed for this configuration, the fact that the time constant of the changing mode is a function of other modes provides a memory capability. Speed of the devices made was about $10^{-11} \mathrm{sec}$, but could be made $10^{-12} \mathrm{sec}$ by decreasing the length of the devices.

\section{Couplers}

Couplers between fibers and thin films are still a problem, ${ }^{4}$ and three different approaches were presented on this subject. R. T. Kersten [WB5] of Siemens described coupling from a film to a multimode fiber: a droplet of high index fluid is applied to the surface in order to make the guide leaky; the fiber then collects the light that is coupled out. For the experiment shown, the waveguide was made by diffusion into Schott FK6 glass, which gives a refractive index of 1.44 for the substrate and 1.47 for the waveguide. The multimode fiber had refractive indices of 1.62 and 1.52 for core and cladding, respectively, 


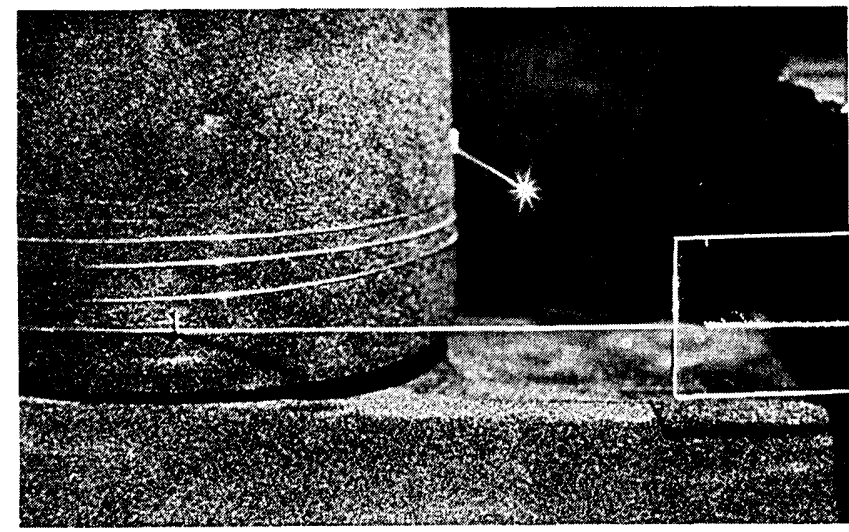

Fig. 2. A film-to-fiber coupler by P. K. Tien, G. Smolinsky, and R. J. Martin [WB6].

Maximum coupling efficiency (about 50\%) depends upon the angle between slab and fiber; optimum angle was $25^{\circ}$ for a fluid with refractive index 1.62, $25.5^{\circ}$ for one with index 1.66. The acceptance angle of the multimode fiber (about $68^{\circ}$ ) allows good coupling from a multimode slab waveguide to a multimode fiber. Good coupling from fiber to monomode slab would require a monomode fiber, and the coupling angle then becomes more critical.

A second film-fiber coupler, described by $G$. Smolinsky et al. of Bell [WB6], utilized a film waveguide with a tapered edge deposited on a substrate with a hole drilled into the end of the substrate perpendicular to the tapered edge to accommodate the fiber (see Fig. 2). The end of the hole is made hemispherical and filled with a liquid of relatively high refractive index to focus into the fiber the light leaking into the substrate from the tapered film. Coupling efficiency is about $50-60 \%$ from film to fiber but is poor in reverse, since the film cannot accept the conical pattern of light coming from the fiber. Attempts will be made to make a flat fiber. The experiments described were with a $0.56-\mathrm{mm}$-diameter fiber. Computer calculations were made to give the pattern of light radiated from the tapered edge into the substrate.

In a third coupler, described by $\mathbf{O}$. Krumpholz and E. Pfeiffer of AEG-Telefunken Research Institute [WB7], the fiber is fixed eccentrically in a connector that may be rotated to bring it into exact alignment with a thin-film device or another fiber. Coupling efficiency as a single-mode fiber-fiber coupler was in the $89-94 \%$ range. It is hoped that it can be used as a universal coupler from laser diode to fiber, from fiber to fiber, or from fiber to thin-film photodiode receiver.

A paper by R. Ulrich [WB3] of Max Planck-Institute für Festkörperforschung demonstrated good frequency selectivity in a prism coupler by adding a semitransparent reflecting coating near the base of the prism. This work has since been published. ${ }^{5}$

R. K. Winn and J. H. Harris [WB8] of the University of Washington presented an analysis for ta- pered horn-shaped couplers from planar film guides to linear waveguides. M. G. F. Wilson and G. A. Teh [WB9] of University College London described a tapered velocity coupler for improving the tolerance in optical directional couplers. If phase matching is attempted with uniform coupled waveguides, tolerances become very difficult as the coupling becomes long. If one guide is tapered, complete power transfer can be effected in the region of approximate phase match if the coupling coefficient and degree of taper are properly chosen. If there is an error that displaces the curve of phase constant vs distance of either guide, the point of crossover simply moves along the guide but full power transfer is still achieved. Preliminary experiments using 7059 glass films sputtered on a glass substrate were described.

\section{Lenses}

J. Harper and E. Spiller [WB11] of IBM described surface lenses made by depressions or protrusions as proposed by Van Duzer ${ }^{6}$ for acoustic waves and Righini et al. ${ }^{7}$ for optics but with a compensation for spherical aberration by filling the spherical depression partially or totally with a dielectric. Figure 3 shows a spherical depression with dielectric coating and the optimum ratio of index in the depression to index outside for various apertures. Photographs of rays focused by the lenses clearly showed the decrease in spherical aberration by adding the dielectric coatings. (See cover!)

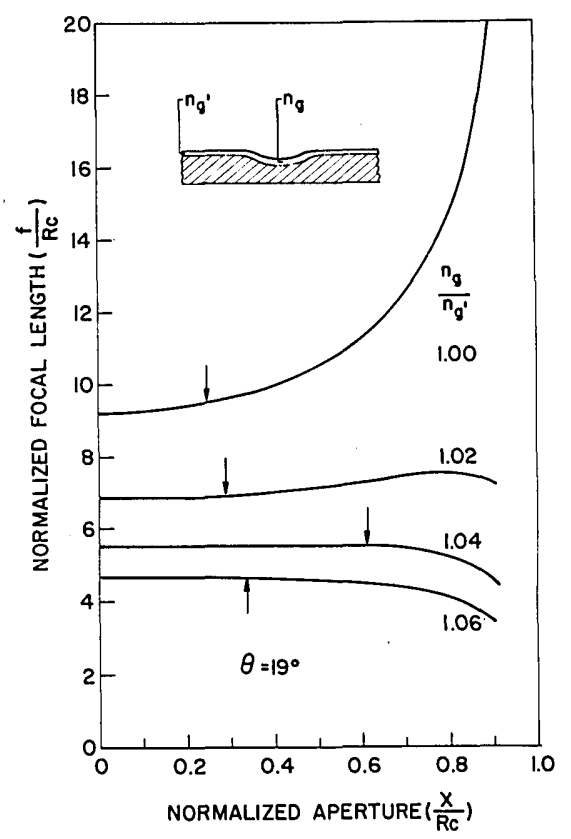

Fig. 3. Spherical-depression lens with dielectric coating described by Harper and Spiller [WB11]. $X$ is the aperture, $R_{c}$ the radius of the circle defining the edge of the lens, and $f$ the focal length. The arrows mark the apertures where the depth of focus is equal to the change in focal length due to the aberrations. 


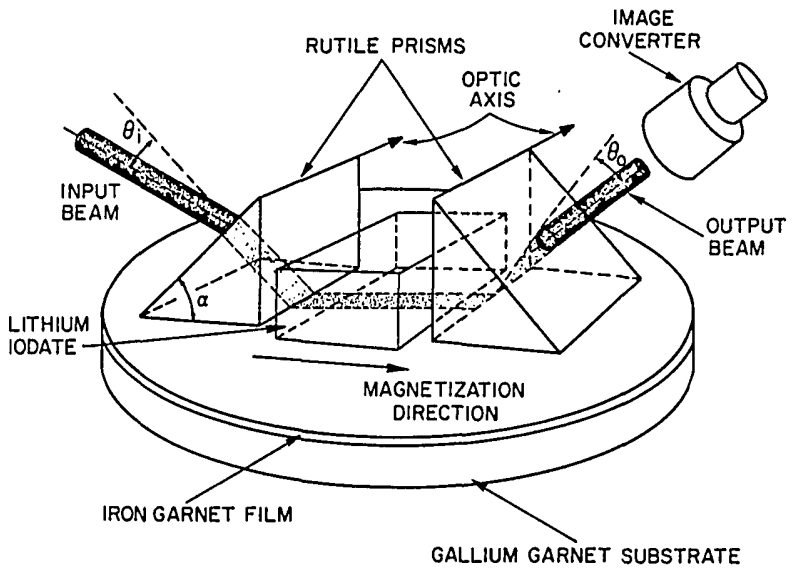

Fig. 4. Configuration of the nonreciprocal optical waveguide described by Warner [MB11]

\section{Unusual Optical Waveguides}

J. A. Arnaud [WB12] of Bell Laboratories demonstrated that single-mode propagation can be obtained in two-dimensional, oversize guides by coupling to a substrate supporting radiation modes. The loss mechanism is analogous to Cerenkov radiation and is that which ensures single-mode propagation in cladded fibers and thin films. The substrate can be a dielectric or a rod supporting whispering-gallery modes; with proper choice of size and curvature it can be of the same material as the guide and in physical contact with it. The coupling theory giving the radiation loss for such coupled systems was recently published by Arnaud. ${ }^{8}$

Very interesting effects in $\mathrm{KNbO}_{3}$ were described by J. R. Tucker [MB12] et al. of The Aerospace Corporation. Naturally occurring sheet domain twins of thickness as low as $\mu \mathrm{m}$ (and probably obtainable to 1 $\mu \mathrm{m}$ ) were observed in crystals of this material grown by modified flux growth techniques from a $\mathrm{K}_{2} \mathrm{O}$ flux. In the twins, two of the crystal axes lie at $45^{\circ}$ to the domain boundaries and flip directions between adjacent domains. Light trapping of $\mathrm{He}: \mathrm{Ne}$ laser beam in those planar domains was observed for several modes. Analysis shows that all the modes are hybrid and that all have small but finite leakage because of the birefringence of the guides. The domains were found to shift in position with pressure, suggesting that they might be useful for modulation or scanning by acoustic signals.

J. Warner [MB11] of the Royal Radio Establishment described work on an isolator for optical waveguides. The configuration (Fig. 4) utilizes a magnetooptic waveguiding film of (LaY) ${ }_{3} \mathrm{Ga}_{0.33} \mathrm{Fe}_{4.67} \mathrm{O}_{12}$, a substrate of $\mathrm{Gd}_{3} \mathrm{Ga}_{5} \mathrm{O}_{12}$, and an anisotropic top layer of $\mathrm{LiIO}_{3}$. The anisotropic top layer is complementary to the magnetooptic film and also makes the TE and TM modes degenerate. The device had not worked at the time of the presentation, presumably because of poor contact between the $\mathrm{LiIO}_{3}$ and the waveguiding film.

\section{Thin-Film Optical Harmonic Generators}

A paper by $\mathbf{N}$. Uesugi et al. [MB10] of Tohoku University described optical second and third harmonic waves generated in oriented polycrystalline thin-film waveguides. Thin-film harmonic generators give high intensity with relatively low total power; they also permit phase matching by choice of film thickness without requiring birefringence. Spatially averaged second-order nonlinear coefficients were calculated for various mode combinations in the scheme $\omega_{1}+\omega_{1} \rightarrow \omega_{2}$ for $z$-axis-oriented polycrystalline films. The experiments were in $\mathrm{ZnS}$ of cubic type because of its large second-order nonlinearity and wide tuning range in the visible. Output from a $Q$-switch Nd:YAG laser with 3-5 kW peak power was coupled to TE modes in the film by a prism coupler at wavelengths $1.064 \mu \mathrm{m}, 1.112 \mu \mathrm{m}$, and $1.123 \mu \mathrm{m}$. Large clear spectra of the second harmonic were observed; with polarization normal to that of the fundamental waves. Third harmonic spectra, smaller and less pure, were also observed for each of the fundamental wavelengths.

\section{Materials \\ P. K. Tien}

\section{Al-Ga-As System}

The first invited paper was given by E. Garmire of Standard Telecommunications Laboratories [TuA1] on $\mathrm{GaAs}$ for monolithic integrated optics. Her paper contained a wealth of information summarizing the work that had been carried on at Caltech for many years. She started by outlining two methods of achieving a $\mathrm{GaAs}$ waveguiding layer on a GaAs substrate: changing the content of free carrier by epitaxial growth, ion implantation, or diffusion; and (2) changing material composition by liquid phase epitaxy, vapor growth, molecular beam epitaxy, or sputtering. She then described the formation of ridged channel waveguides by ion milling, chemical etch, sputter etch, or growth through mask, and imbedded channel waveguides by ion implantation, diffusion, and refill around ridged waveguides. Excellent directional couplers were formed by using imbedded waveguides. Finally, she discussed a recent experiment at Caltech-an optically pumped distributedfeedback GaAs laser-and concluded that a corrugated feedback injection laser should be possible after the problem of regrowth on a corrugated layer is solved.

A paper on Cr-doped GaAs epitaxial structures for device applications at $10.6 \mu \mathrm{m}$ was reported by J. F. Black, J. L. Swindal, and P. K. Cheo [TuA3] of United Aircraft Laboratories. Waveguiding epitaxial layers were formed by reducing the free concentration $1 \times 10^{17}$ to $2 \times 10^{18} \mathrm{~cm}^{-3}$ of the substrates to a concentration of $10^{12} \mathrm{~cm}^{-3}$. These epitaxial layers have a perfect mirrorlike surface and can have a surface area as large as $3 \mathrm{~cm} \times 5 \mathrm{~cm}$. A technique was also developed for forming grating structures by rf sputter etching over $\mathrm{SiO}_{2}$ mask. 
Another paper on epitaxial GaAs-(GaAl)As layers was presented by S. Kamath [TuA1] of Hughes Research Laboratories. To stabilize the $\mathrm{Al}$ concentration during the growth of large area layers, an infinite-melt liquid epitaxial system was developed that allows resaturation of the melt at intervals.

\section{Rare-Earth Garnet Films}

The use of garnets for waveguides, lasers, and magnetooptical modulators was reported in an invited paper by P. K. Tien [TuA2] of Bell Laboratories. $\mathrm{He}$ indicated that the magnetic iron garnets are transparent only in the spectrum region between 1.1-5 $\mu \mathrm{m}$. These magnetooptic garnets have become important because of the recent development of single-material fibers that have very low losses at 1.15 $\mu \mathrm{m}(2-3 \mathrm{~dB} / \mathrm{km})$.

The magnetic domains are difficult to observe when the magnetization is in the plane of the film. By using a specially constructed microscope, Tien and his colleague were able to photograph these domains, and showed a photograph of periodic domains that have never been observed before. He discussed motion of the magnetization, processes of optical switching, and a magnetooptical switch that can be used for baseband modulation up to $300 \mathrm{MHz}$.

\section{II-VI Compound Waveguides}

W. M. Caton, W. E. Martin, D. B. Hall, and H. F. Taylor [TuA4], all of the Naval Electronics Laboratory Center, reviewed their work on diffused $\mathrm{CdS}_{x} \mathrm{Se}_{1-x}$ waveguides and modulators. Channel waveguides several microns thick have been formed by diffusion through a $\mathrm{SiO}_{2}$ mask. A refractive index difference $\Delta n=0.02$ can be achieved by heating CdS substrate in $\mathrm{S}_{2}-\mathrm{Se}_{2}$ atmosphere at $800^{\circ} \mathrm{C}$ for $3 \mathrm{~h}$. The diffusion constant is calculated to be $10^{-11}$ $\mathrm{cm}^{2} / \mathrm{sec}$.

A paper on evaporated CdS film to form waveguides and detectors was reported by Glasgow University's C. R. Stanley and W. Duncan [TuA7]. They used a 7-W electron gun for evaporation at a substrate temperature of $100^{\circ} \mathrm{C}$ and a background pressure of $10^{-8}$ Torr. The films formed have a loss of $5 \mathrm{~dB} / \mathrm{cm}$. By depositing the CdS film on a Si substrate, they were able to form a CdS-Si junction photodetector that has a response $1 \mathrm{~V} / \mathrm{mW}^{-1}$.

\section{Liquid Crystal Films}

The development of liquid crystal optical waveguides was reviewed in an invited paper by $\mathbf{J}$. $\mathbf{P}$. Sheridan [TuA5] of the Naval Research Laboratory. $\mathrm{He}$ discussed dielectric effects that are the result of a uniform change in direction of optical axis in nematic liquids or the reordering of the optical axis or a change in helix pitch in cholesteric liquids. The application of an electric field acts on the dielectric anisotropy of the fluid and produces an orienting torque. Removal of the field allows the original orientation or structure to be restored. Using these effects, he discussed the experiments of optical switches, electrode prisms, and mode suppression using liq- uid crystal either as the waveguide or as the substrate.

The scattering loss in nematic liquid crystal optical waveguides was discussed in a paper by C. Hu (MIT) and J. R. Whinnery (Berkeley) [TuA6]. Both their calculations and experiments indicate a loss above 20 $\mathrm{dB} / \mathrm{cm}$. However, a loss less than $1 \mathrm{~dB} / \mathrm{cm}$ was observed by Sheridan [TuA5] above. The problem is not fully understood at this time.

\section{Single Crystalline Sillenite Films}

A. A. Ballman, H. Brown, P. K. Tien, and R. J. Martin of Bell Laboratories [TuA8] reported the growth of single crystalline sillenite films by a liquid phase dipping method. The sillenite family involves a group of compounds of $\mathrm{Bi}_{2} \mathrm{O}_{3}$ with $\mathrm{GeO}_{2}, \mathrm{SiO}_{2}$, $\mathrm{TiO}_{2}, \mathrm{ZnO}, \mathrm{Ga}_{2} \mathrm{O}_{3}, \mathrm{Al}_{2} \mathrm{O}_{3}, \mathrm{Fe}_{2} \mathrm{O}_{3}$, and $\mathrm{P}_{2} \mathrm{O}_{5}$. Any of these compounds can be used as the film with any other compound as the substrate. These films have piezoelectric, electrooptic, nonlinear optic, and pyroelectric properties. They also have a large optical activity.

\section{Nonoxide Chalcogenide Glass Waveguides}

Sputtered $\mathrm{Ge}_{28} \mathrm{Sb}_{12} \mathrm{Se}_{60}, \mathrm{Ge}_{33} \mathrm{As}_{12} \mathrm{Se}_{55}$, and $\mathrm{As}_{2} \mathrm{~S}_{3}$ nonoxide chalcogenide glass films were discussed by R. K. Watts and J. R. McIntier of Texas Instruments [TuA9]. These films have a very high refractive index, 2.5-2.8. The loss of chalcogenide glass waveguides can be as low as $2 \mathrm{~dB} / \mathrm{cm}$ at $1.0 \mu \mathrm{m}$.

\section{$\mathrm{LiNbO}_{3}-\mathrm{LiTaO}_{3}$ Solid-Solution Films}

A new $\mathrm{LiNbO}_{3}-\mathrm{LiTaO}_{3}$ solid-solution film grown by a method known as epitaxial growth by melting was reported in Tien's invited paper and later also in Ballman's paper. The film has a transition region between the film and the substrate that has a graded composition, so that any effect of lattice-constant mismatch is minimized. The refractive index profile of the film follows closely a Fermi distribution function. The film was reported to form excellent electrooptic waveguides. In a postdeadline paper, another $\mathrm{LiNbO}_{3}-\mathrm{LiTaO}_{3}$ film was reported, by $\mathbf{W}$. Philips and J. M. Hammer RCA Laboratories. They used a different method: metallic $\mathrm{Nb}$ was first sputtered on a $\mathrm{LiTaO}_{3}$ substrate and $\mathrm{LiNbO}_{3}$ substrate. Excellent electrooptical modulators were reported by using these films.

\section{Periodic Structures and Gratings E. M. Conwell}

The papers of this group are linked by the principle that a small periodic perturbation of the properties of a waveguide can couple two guide modes of the unperturbed guide with propagation vectors $\beta_{l}$ and $\beta_{0}$, respectively, provided

$$
\beta_{l}-\beta_{0}=2 \pi l / \Lambda, \quad l=1,2, \ldots,
$$

$\Lambda$ being the spatial period of the perturbation. Coupling is strongest for $l=1$, and in what follows we shall assume this to be the case unless otherwise specified. 


\section{Coupled-Mode Formalism}

In an invited paper that established the theoretical background for a number of succeeding papers, $\mathbf{A}$. Yariv of Caltech [TuB1] described his recent work ${ }^{9}$ in applying the coupled-mode formalism to integrated optics. This formalism is widely used. Yariv pointed out that it provides a uniform way of describing a wide range of phenomena, including those that arise from a periodic perturbation: distributed feedback (DFB), transfer of energy from one mode to another, optical filtering and reflection, etc. The same formalism also describes electrooptic, acoustooptic, and magnetooptic modulation; second harmonic generation; and wave coupling in devices such as directional couplers. For small perturbations of any of the above kinds that link two modes, the theory leads to a pair of coupled first-order differential equations, each describing the rate of growth of one of the modes as a linear function of the amplitude of the other with a coupling parameter that is determined by the detailed nature of the perturbation. When the above equation is not satisfied exactly, there is an additional exponential factor present that limits the energy transfer and causes it to oscillate in time. The presence of gain or loss in the medium gives rise to additional terms in the differential equations. In the talk the theory was applied mainly to the case of DFB lasers, in which a forward wave with $\beta_{l}$ is strongly coupled by a periodic perturbation to a backward wave with $\beta_{0}=-\beta_{l}$, making mirrors unnecessary. Although proceeding somewhat differently, the theory was shown to lead to results equivalent to those of Kogelnik and Shank. ${ }^{10}$ Using the theoretically derived coupling constant $k$ for the practically important case of DFB due to a corrugation of amplitude $a$ on the guiding layer, Yariv noted that for $a \ll$ $\lambda_{0}$, the free space wavelength of the light, the coupling is independent of the size of the difference between the indices of the corrugated guiding medium and the adjacent medium. Thus, for example, in a three-layer structure of $\mathrm{Ga}_{x} \mathrm{Al}_{1-x} \mathrm{As}, \mathrm{GaAs}$, and air the same effect could be obtained by corrugating either surface of the GaAs, provided, of course, $a \ll \lambda_{0}$.

\section{Filters}

In the talk that followed, D. C. Flanders, R. V. Schmidt, and C. V. Shank of Bell Labs. [TuB2] reported on the fabrication of reflection filters based on the strong conversion from a forward mode to a backward mode of the same $|\beta|$ by means of a periodic corrugation. The center wavelength of the filter is that at which the above condition is exactly satisfied with $l=1$ and its fractional bandwidth is approximately $\Lambda / L$, where $L$ is the length of the corrugated region. The filters were fabricated on single-mode sputtered glass waveguides by coating the waveguide with photoresist, interferometrically exposing it with a $3250-\AA$ $\mathrm{HeCd}$ laser, and then ion-beam etching it. As determined with the use of a narrow line $(<0.25 \AA)$ tunable dye laser, bandwidths of less than $2^{\circ}$ and efficiences over $75 \%$ were achieved. The filter may be tuned over hundreds of angstroms by changing the incident angle of the input light with respect to the grating.

\section{Energy Transfer between Modes}

F. W. Dabby and A. Kestenbaum of Western Electric [TuB3] reported on experiments with corrugated photoresist guides having $\Lambda$ 's large enough that coupling (or scattering) was possible only between modes propagating in the same direction. They demonstrated energy transfer from one guide mode $(m=1)$ to another $(m=0)$ and attenuation of the $m$ $=0$ mode due to coupling into substrate modes. These results demonstrate the possibility of obtaining low frequency cutoff filters.

Coupling into substrate modes was also demonstrated in a postdeadline paper by $\mathbf{R}$. Albanese and C. F. Quate of Stanford University. In this case, however, the coupling was achieved by acoustic surface waves with wavelength $\Lambda$ satisfying the above condition. The waveguide was an out-diffused $\mathrm{LiNbO}_{3}$ crystal, $^{3}$ and the surface waves, with a frequency of $116 \mathrm{MHz}$, were produced by interdigital transducers on the $\mathrm{LiNbO}_{3}$ surface. The unguided mode was found to emerge at an angle of $7.66^{\circ}$. This coupling scheme had been proposed earlier by Oliner, ${ }^{11}$ who pointed out its merits for wide-angle laser scanning.

\section{Distributed-Feedback Lasers}

Shyh Wang of UC Berkeley [TuB4] presented a theoretical treatment of thin-film lasers with periodic structures that may exist beyond the active, or gain, region as well as within it. Threshold gain, frequency control, and mode selectivity were analyzed for two extreme cases: that in which the periodic structure is confined to the active region, i.e., the DFB laser, and that in which the periodic structure is limited to regions bordering on the active region, called the distributed Bragg-reflector (DBR) laser. Applying the results to GaAs, with values assumed for the coupling constants that should be attainable, Wang concluded that the DBR laser could have a lower threshold gain than the DFB laser or than a conventional cavity laser. In addition, he finds that mode discrimination, isolation from the outside circuit, flexibility for pumping, and modulation should all be better for DBR lasers than DFB ones.

Wang's theory allowed for one constant grating spacing inside the gain region and different ones on either side of the gain region. In the paper that followed, R. Shubert of Rockwell [TuB5] considered more general DFB structures, allowing discontinuously varying regions in gain, coupling, and propagation constant. As an example, he used a periodic structure with period $\Lambda$ that covers both ends of the gain region but not the center, the gap $l$ between the two periodic sections taken to be an integer times $\Lambda$. Coupled-mode theory is used to analyze these structures, the matching of solutions at the discontinuities giving eigenvalue equations that yield (through nu- 
merical solution) the lasing frequencies and threshold gain characteristics. For the example cited, propagation constants in the corrugated and noncorrugated regions are allowed to differ by $\Delta \beta$. For $\Delta \beta l$ $=(n+1 / 2) \pi, n$ being an integer, Shubert finds the mode spectrum to be symmetrical about the Bragg frequency $\omega_{0}$, with the lowest order resonance occurring at $\omega_{0}$, in contrast to the case of a continuous grating with $\Delta \beta=0 .{ }^{10}$ For values of $\Delta \beta l=(n+1 / 2) \pi$ or $n \pi$ the spectrum becomes asymmetrical with respect to $\omega_{0}$ in both frequency and threshold gain. The use of nonuniform periodic structures, asymmetrical in gain or coupling, was shown to offer opportunities for lowering threshold gain for the lower modes and simultaneously increasing it for the higher order modes.

C. Elachi and G. Evans, of Caltech, and C. Yeh of UCLA [TuB6] extended the theory of the DFB laser ${ }^{10}$ by allowing for (1) the propagation vector having a component perpendicular to the propagation direction as well as parallel to it; (2) the reflected energy going into a different mode than the original incident mode; (3) the gain being in the substrate rather than in the thin film. They assumed sinusoidal surface corrugation and found that the coupling coefficient for this case depends on the size of the field at the boundaries of the guide. As a consequence, threshold gain for the lowest modes was found to be very large at low frequencies (near cutoff), to decrease with increasing frequency, and finally to increase again for high frequencies (guide width $\gg \lambda_{0}$ ). For the same reason they found that over a wide range of frequencies, for a given input mode, threshold gain is lower when the output mode is of higher order than the input mode.

Analyzing the transmission and reflection properties of DFB lasers, with the theory of Kogelnik and Shank ${ }^{10}$ as the starting point, S. R. Chinn and P. L. Kelley [TuB7] MIT derived a simple relation between gain and linewidth of a DFB amplifier near oscillation threshold. This relation and the general methods of noise theory, plus some simplifying assumptions, led to a theoretical expression relating bandwidth and output power of the amplified spontaneous emission in the $n$th mode.

Recently, GaAs waveguides with feedback arising from surface corrugations have been made to lase under optical pumping. ${ }^{12}$ It is desirable to make an injection laser with DFB because a grating structure, which could be made with precise repeat distance by optical techniques, for example, could pin the frequency down to a narrow predictable line, which the present heterostructure does not. In addition, the corrugation process should be compatible with conventional integrated circuit technology. In a postdeadline paper X. Yang and X. Ballantyne of Cornell reported on the successful growth of a highly doped $p$-type GaAs epitaxial layer over the corrugated surface of an $n$-type substrate using solution epitaxy. The grating on the substrate was prepared using a scanning electron microscope to make the patterns and then ion-beam machining it. Grating periods were $\sim 350 \mathrm{~nm}$ and depths $\sim 100 \mathrm{~nm}$. The junction displayed good electrical properties. The growing of a $\mathrm{Ga}_{0.5} \mathrm{Al}_{0.5} \mathrm{As}$ layer over a periodically etched GaAs layer was reported by Shank [MB1] above. That structure lased under optical pumping.

Theoretical considerations relating to the optimization of GaAs-GaAlAs injection lasers with internal corrugation feedback were presented in a paper by M. Nakamura (Hitachi) A. Yariv (Caltech) [TuB9]. They pointed out that a double heterostructure where feedback is obtained with corrugations produced by ion-milling the active layer interface would have high threshold current density due to nonradiative recombination arising from milling damage. To overcome this they proposed a separate confinement heterostructure in which the carriers are confined in a very narrow $p$-GaAs layer and the electromagnetic wave is confined to this layer plus wider surrounding layers of $p$ - and $n-\mathrm{Ga}_{1-y} \mathrm{Al}_{y} \mathrm{As}$. The corrugation is to be between the $p-\mathrm{Ga}_{1-y} \mathrm{Al}_{y} \mathrm{As}$ and an adjacent, lower index $p-\mathrm{Ga}_{1-x} \mathrm{Al}_{x} \mathrm{As}$ layer, thus sufficiently separated from the gain region to eliminate the effects of milling damage. The threshold behavior of a laser based on this structure is analyzed theoretically using the theoretical coupling constant discussed earlier by Yariv [TuB1] and the theory of Kogelnik and Shank. ${ }^{10}$ For numerical evaluation the parameters assumed were corrugation height of $500 \AA$ and $\Lambda=$ $0.11 \mu \mathrm{m}$, required for $l=1$ in the equation. The theory predicts that the required threshold gain decreases as the thickness $t$ of the light-confining layer decreases, as expected, since the coupling is inversely proportional to $t^{3}$. Below $t \simeq 1 \mu \mathrm{m}$ the threshold gain is found to drop sharply. In addition for short (length $<0.5 \mu \mathrm{m}$ ), thin (thickness $<1 \mu \mathrm{m}$ ) lasers the threshold gain of the corrugated laser is predicted to be much lower than that of its conventional FabryPerot counterpart.

\section{Grating Couplers}

Two theoretical papers on grating couplers, pointing out that previous studies have employed approximations that are two restrictive for many practical cases, gave rigorous treatments. S. T. Peng, T. Tamir, and H. L. Bertoni [TuB8] of PINY treated the case of a grating with alternating rectangular regions of real dielectric constants $\epsilon^{\prime} \neq \epsilon^{\prime \prime}$, with corresponding widths $d^{\prime}$ and $d^{\prime \prime}$, respectively. M. Cadilhac, M. Neviere, P. Vincent, and $R$. Petit of Lab. Optique Electromagnetique [TuB11] allowed the grating to have an arbitrary shape, its height $y$ at the position $x$ being given by $y=f(x)$, with $f(x)$ a periodic function. In both papers it was considered that the structure consisted of four layers: ambient, grating, guiding film, and substrate. Because of grating periodicity, waves guided by the structure consist of an infinite set of partial fields, each propagating along $x$ with wavenumbers $\beta_{l}$ satisfying the above equation, $l$ now being permitted to take on the value 0 as well as $\pm 1, \pm 2$, etc. The form of the solutions of the wave equation for the homogeneous regions is well known. For the inhomogeneous region contain- 
ing the grating Cadilhac et al. emphasized strongly that the solution can only be approximated by a Rayleigh expansion (series of progressing and evanescent plane waves) when the grating height is quite shallow compared to the period. They present a systematic method of solution for the wave equation in this region that involves numerical integration. For the inhomogeneous region, Teng et al., take advantage of known solutions of the wave equation for an unbounded medium with alternating regions of $\epsilon^{\prime}$ and $\epsilon^{\prime \prime}$ as in the grating structure. Application of the usual boundary conditions of $E$ and $H$ at the three boundaries involved in the four layers leads to an infinite set of homogeneous equations for the amplitudes of the partial waves. The condition that these lead to a nontrivial solution, plus suitable truncation, yields the values of $\beta$ for $l=0$ of the guided waves. These values are in general complex, the imaginary part $\alpha$ determining the rate of energy leakage out of the guided wave and thus, essentially, the efficiency of the grating. From calculations of $\alpha$ for a configuration with $\epsilon^{\prime \prime}$ equal to the dielectric constant of the ambient, $\epsilon^{\prime}$ less than the dielectric constant of the film, Teng et al. find that as the grating thickness increases from $0, \alpha$ and thus the coupling increase but then level off. Increasing the height of the teeth beyond this point will not increase coupling. It may, however, be increased by increasing $\epsilon^{\prime}$. As Teng et $a l$. point out, their analysis gives an accurate and systematic procedure for developing practical design criteria for gratings with rectangular geometry. The formalism of Cadilhac et al. should accomplish this for gratings of other shapes.

\section{Diffractors and Modulators}

\section{A. Yariv}

Waveguide modulators, in which the same material doubles as the guiding layer and the modulation medium, promise to lead to orders of magnitude reduction in modulation powers compared to their bulk counterparts.

One such modulator was described in a postdeadline paper by W. Phillips and J. M. Hammer of RCA Laboratories. The waveguiding takes place in a $\mathrm{LiNb}_{x} \mathrm{TaO}_{3}$ layer on a $\mathrm{LiTaO}_{3}$ substrate. The layer is produced by a diffusion of metallic $\mathrm{Nb}$ into the $\mathrm{LiTaO}_{3}$ substrate. An attractive feature of this method is the small height $(<2 \mu \mathrm{m})$ of the guiding layer, which leads to reduced modulation powers. Bragg coupling-type modulators using these waveguides required $6.5 \mathrm{~V}$ for $80 \%$ modulation with an estimated $0.5 \mathrm{~mW}$ modulation power per $\mathrm{MHz}$.

F. K. Reinhart [WA6] of Bell Laboratories reported on a waveguide modulator using $\mathrm{Al}_{x} \mathrm{Ga}_{1-x} \mathrm{As}-$ $\mathrm{Al}_{y} \mathrm{Ga}_{1-y} \mathrm{As}-\mathrm{Al}_{z} \mathrm{Ga}_{1-z} \mathrm{As}$ sandwiches. The inner layer has a thickness $0.1 \mu \mathrm{m}<W<1 \mu \mathrm{m}$ with $y<$ $x, z$. this extreme confinement, used successfully in reducing the threshold of double heterostructure junction lasers, leads to extremely low modulation powers.

The following specifications and data relevant to the modulator were given for $\lambda=1.06 \mu \mathrm{m}$ :

$\begin{array}{lc}2 \mathrm{~W}(\mu \mathrm{m}) & 0.5 \\ \text { Length }(\mathrm{mm}) & 0.83 \\ \text { Width }(\mu \mathrm{m}) & 50 \\ \text { Halfwave voltage }(\mathrm{V}) & 5.9 \\ \Delta \phi_{0}(\text { degree } / \mathrm{V} \mathrm{cm}) & 370 \\ P_{0}\left[\mathrm{mw} / \mathrm{MHz}(\mathrm{rad})^{2}\right] & 0.08 \\ C(\mathrm{pf}) & 15\end{array}$

Such waveguide modulators can be produced monolithically with GaAs DH lasers and are thus interesting for potential integrated-circuit applications.

GaAs modulators for $10.6-\mu \mathrm{m}$ lasers were described by J. F. Lotspeich [WA4] of the Hughes Research Laboratories. The guiding layer was a mechanically polished GaAs platelet ( $\sim 25 \mu \mathrm{m}$ thick) with bounding layers of insulating CdTe or $\mathrm{As}_{2} \mathrm{~S}_{3}$. The use of low loss layers, which distinguishes this work from previously reported work, leads to propagation losses as low as $0.7 \mathrm{~dB} / \mathrm{cm}$ for the $m=0 \mathrm{TE}$ mode. The corresponding half voltage was $480 \mathrm{~V}$. Lotspeich described plans for $200-\mathrm{MHz}$ modulators based on this technique.

Theoretical considerations and preliminary data dealing with light deflection in single and multimode waveguides using acoustooptic interactions were described by J. F. Weller, T. G. Giallorenzi, and A. F. Milton [WA9] of the Naval Research Laboratory.

Acoustooptic scanners based on a similar principle were described by F. R. Gfeller [WA11] of University College London and in a postdeadline paper by $\mathbf{R}$. Albanese and C. F. Quate of Stanford University. In the Stanford experiment the interaction takes place between a surface acoustic wave and a confined optical mode both traveling colinearly in an out-diffused $\mathrm{LiNbO}_{3}$ waveguide.

The acoustic strain wave couples power from the confined waveguide mode into a radiation mode traveling at a finite angle to the waveguide axis. This angle of deflection is a function of the acoustic frequency. A change of frequency from $100 \mathrm{MHz}$ to 150 $\mathrm{MHz}$ should result in one hundred resolvable spots. The transducer used in the reported experiment made possible an acoustic frequency tuning of 10 $\mathrm{MHz}$.

\section{Fabrication of Integrated Optical Circuits \\ A. J. De Maria}

Ten papers, including two invited papers, dealt with the most important aspect of integrated opticsoptical integrated circuits and waveguide fabrication techniques. Basic techniques reported at this meeting involve the use of electron and ion-beam lithography, which differs very little from those reported two years ago at the First Topical meeting, held in Las Vegas. The only notable difference is in the degree of sophistication of controls needed for the delineation of complex optical circuits. D. B. Ostrowsky [ThAl] of Thomson-CSF described a computerized electron microscope with an interferometrically controled positioning of the substrate. Circuit elements including lines, circles, squares, and triangles were made with edge definition better than $500 \AA$. A du- 
plication technique has been developed that permits rapid fabrication of masks and circuits with a $2000-\AA$ precision maintained over a $5-\mathrm{cm} \times 5-\mathrm{cm}$ substrate surface area.

H. L. Garvin and E. D. Wolf [ThA6] of Hughes Research Laboratories elaborated on the use of ion milling to produce a good facsimile of the delineating mask made with a scanning electron beam. Threedimensional waveguides with an edge definition better than $200 \AA$ have been fabricated. Parallel channels at a separation of $0.5 \mu \mathrm{m}$ to permit interchannel coupling and grating couplers with a period as small as $1150 \AA$ have been produced.

Of particular interest were papers [ThA3] and [ThA4] describing a convenient way to obtain lowloss optical waveguides with excellent electrooptic, acoustooptic, and nonlinear optic properties by either out-diffusion or in-diffusion methods. When $\mathrm{LiNbO}_{3}$ of $\mathrm{LiTaO}_{3}$ are heated in a vacuum, $\mathrm{Li}_{2} \mathrm{O}$ evaporates from the surface and additional $\mathrm{Li}_{2} \mathrm{O}$ diffuses up to the surface from the interior. By diffusion and evaporation, a significant refractive index increment is produced within a depth that varies from a few microns to tens of microns below the surface. To some extent the guiding layer thickness can be controlled by controling the time and temperature. J. M. Hammer and W. Phillips of RCA in a postdeadline paper reported the production of a lowloss $\mathrm{LiNb}_{x} \mathrm{Ta}_{1-x} \mathrm{O}_{3}$ optical waveguide by diffusion of metallic niobium film into the $\mathrm{LiTaO}_{3}$ substrate. The effective thickness and index can be controled to produce single-mode waveguides. By this method, the index increment in the guiding layer is about ten times larger than that obtainable by the out-diffusion method. Typical penetration depth of $\mathrm{Nb}$ is about 1 $\mu \mathrm{m}$. Active thin-film devices such as the electrooptic Bragg switch ${ }^{13}$ or phase modulator ${ }^{14}$ can be made by utilizing this waveguide.

Most of the credit for the success of this meeting goes to P. K. Tien, the chairman of the Program Committee, for the well balanced technical content of the meeting and to OSA for planning and organiza- tion. A great deal of credit goes also to some dozen members of the Program Committee, several of whom are also the coauthors of this report, for their invaluable contributions in shaping up in the program. This review was edited by R. V. Pole, who chaired the overall meeting.

A Digest of this meeting giving extended summaries of papers may be obtained from the Optical Society of America, 2000 L Street, N.W., Washington, D.C., 20036 , for $\$ 15.00$.

The Program Committee for the meeting consisted of R. V. Pole, Chairman (IBM Research); P. K. Tien, Program Chairman (Bell Laboratories); E. Ash (University College London); P. Burlamachi (Instituto di Ricerca Sulle Onde Electromagnetiche; A. J. DeMaria (United Aircraft Research Laboratories); V. Evtuhov (Hughes Research Laboratory); J. H. Harris (University of Washington); E. Lean (IBM Research); S. E. Miller (Bell Laboratories); H. Sasaki (Fujitsu Laboratories); E. Spitz (Thomson-CSF); A. Yariv (Caltech).

\section{References}

1. S. E. Miller, E. A. J. Marcatili, and T. Li, Proc. IEEE 61, 1703 (1973).

2. G. Zeidler and D. Schicketanz, Siemens R \& D Rept. 2, 227 (1973).

3. J. Nishizawa, IEEE J. Quantum Electron. QE-4, 143 (1968).

4. See, for example, L. P. Boivin, Appl. Opt. 13, 301 (1974).

5. R. Ulrich, Appl. Phys. Lett. 24, 21 (1974).

6. T. Van Duzer, Proc. IEEE 58, 1230 (1970).

7. G. C. Righini, V. Russo, S. Sattini, and G. Toraldo di Francia, Appl. Opt. 11, 1442 (1972).

8. J. Arnaud, Bell Syst. Tech. J. 53, 217 (1974).

9. A. Yariv, IEEE J. Quantum Electron. QE-9, 919 (1973).

10. H. Kogelnik and C. V. Shank, J. Appl. Phys. 43, 2237 (1972).

11. A. A. Oliner, Annual Review of Joint Services Electronic Program, Polytechnic Institute of Brooklyn, 3-4 Dec. 1968.

12. H. W. Yen, M. Nakamura, E. Garmire, S. Somekh, A. Yariv, and H. L. Garwin, Opt. Commun. 9, 35 (1973).

13. J. M. Hammer, D. J. Channin, and M. T. Duffy, Appl. Phys. Lett. 23, 176 (1973).

14. I. P. Kaminow, J. R. Carruthers, E. H. Turner, and L. W. Stulz, Appl. Phys. Lett. 22, 540 (1973).
CONTINUING ENGINEERING EDUCATION

300 CHRYSLER CENTER - NORTH CAMPUS

THE UNIVERSITY OF MICHIGAN

ANN ARBOR, MICHIGAN 48105

\section{A N N O U N C I N G}

197.5 ENGINEERING SUMMER CONFERENCES

\section{COHERENT OPTICS}

Chairman: Emmett N. Leith

August 4-8, $1975 \quad$ Fee: $\$ 350$

Presents physical optics from the Fourier

viewpoint. Applications such as spatial

filtering, interferometric metrology and

holography are covered. Practical experience

is offered through laboratory sessions, where

participants will perform experiments. 